\title{
A Brief Introduction of Qigong Dynamic Qigong: Tai Chi
}

\author{
Jeff Chui* \\ Lecturer of Sports and Physical Education, Hong Kong Baptist University, Hong Kong
}

*Corresponding author: Jeff Chui, Lecturer of Sports and Physical Education, Hong Kong Baptist University, Hong Kong.
Received Date: March 05, 2019

Published Date: March 19, 2019

\section{What is Qigong?}

Our body has a meridian network where an energy called "Qi" circulates. The flow of Qi correlates to our health and well being. Qigong is a methodology for promoting smooth flow and maintaining a balance of Qi within the meridian network.

But why do we care about Qi flow? As there are strong correlation between Qi flow in the meridians and healthiness of our internal organs, we can practice Qigong as a self-healing, natural therapy to health issues and a preventive measure.

Qigong could generally be categorized into two major groups, static and dynamic. Static Qigong's characteristics are nearly motionless, maintain a posture such as sitting or standing for an extended period of time. Dynamic Qigong, on the other hand, consists of continuous movements, emphasize on the change of postures and movements. As in the theory of Yin Yang (there is Yin within Yang, and Yang within Yin, see Figure 1), there is no absolute Static nor Dynamic Qigong as Static Qigong has some motions and Dynamic Qigong has its quiet time. Tai Chi and Yoga are good examples of Dynamic Qigong, while Tai Chi is more dynamic relative to yoga.

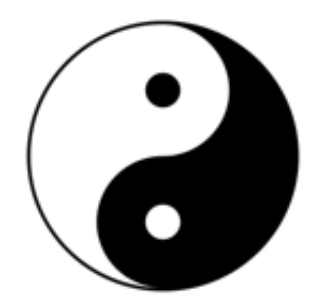

Figure 1: There is Yin within Yang, and Yang within Yin, see figure 1

\section{Practicing Tai Chi as a form of Dynamic Qigong}

Tai Chi can be used as a daily exercise to enhance body balancing and tranquillizing the mind. However, to maximise the benefits, it should be learnt and advanced through 6 stages, namely form, posture, mindfulness, jin (an internal force), Qi, and spiritual:
a. Form: Perform the Tai Chi movements
b. Posture: Perform the form at precise positioning and in continuous and body synchronization
c. Mindfulness: Highly concentrate in performing the movements but at ease; instinctively

d. Jin: The movements are smooth and effortless, yet the body is filled with an internal force

e. Qi: The mind commands the Qi to flow, and Qi drives the body as each movement is performed

f. Spiritual: The previous five levels are fused together in every movement

While each stage is more advanced than the previous stage, the previous stage is fundamental to the next stage, especially stage 6, spiritual cannot be achieved without the solid foundation of each of the first five stages. In fact, Qi flow is already enhanced even at stage 1, making Tai Chi a great form of Dynamic Qigong.

If Tai Chi has it all, why bother with Static Qigong? Although Tai Chi is suitable for all age groups, it does require efforts to memorize the movements, such that one can reach the gating stage which is mindfulness. Since Static Qigong usually involves a lot less movements and posture requirements, it is easier for one to reach the mindfulness stage and advance directly into the Qi stage.

\section{Acknowledgement}

None.

\section{Conflict of Interest}

No conflict of Interest. 